\title{
Some important considerations in analysis of earthquake-induced landslides
}

\author{
Brian Carlton*, Amir M. Kaynia and Farrokh Nadim
}

\begin{abstract}
Background: The frequency of landslide disasters is increasing as a result of exploitation of natural resources, deforestation, and greater population vulnerability due to growing urbanization and uncontrolled land-use. Earthquakes are a major triggering factor of landslides, and earthquake-induced landslides pose a major threat to infrastructure and human life. This paper presents the effects of slope angle, soil sensitivity, ground motion orientation, and multidirectional shaking on the results of seismic slope stability analyses.

Results: The results show that permanent shear stresses due to sloping ground, strain softening and sensitivity, ground motion orientation, and multidirectional shaking all have a large influence on the permanent displacements estimated from earthquake-induced landslide hazard analyses. Multidirectional shaking also predicts larger excess pore pressures in deep layers than unidirectional shaking.

Conclusions: It is important that site investigations provide adequate information to model the correct slope angle and soil sensitivity. The ground motion orientation should be considered and chosen based on the specific needs of a project. Analyses with only one ground motion component could give unconservative results.
\end{abstract}

Keywords: Slope stability, Earthquake, Soil sensitivity, Strain softening, Multidirectional shaking

\section{Background}

Earthquakes are a major triggering factor of landslides. This was highlighted by the 25 April 2015 Gorkha, Nepal earthquake sequence. The earthquake and its aftershocks killed nearly 9,000 people and triggered thousands of landslides. The landslides were responsible for hundreds of those deaths, blocked vital road and lifeline routes to villages, and created temporary dams across several rivers that were a major concern for villages located downstream. The earthquake-induced landslides continue to pose an immediate and long-term hazard to people and infrastructure within central Nepal (Collins and Jibson, 2015).

This event and similar recent earthquakes, such as the Wenchuan earthquake of 12 May 2008 and the Kashmir earthquake of 8 October 2005, have underscored the threat that earthquake-induced landslides represent to human life, property, built environment and infrastructure in mountainous and hilly regions of the world. The frequency of landslide disasters is increasing as a result of exploitation of

\footnotetext{
* Correspondence: brian.carlton@ngi.no

Norwegian Geotechnical Institute, Postboks 3930 Ullevål Stadion, 0806 Oslo, Norway
}

natural resources, deforestation, and greater population vulnerability due to growing urbanization and uncontrolled land-use. For example, traditionally uninhabited areas such as mountains are increasingly used for recreation and transportation purposes, and new technologies for resource extraction are pushing the borders further into more hazardous areas. This has stimulated more research on earthquake-induced landslides and methods of prediction, mapping and mitigation.

Traditionally, geotechnical engineers have assessed the seismic stability of slopes by pseudo-static analysis where the inertial force caused by ground acceleration is applied as an effective static load equal to the mass of the soil times the peak or effective acceleration. However, the duration of the peak earthquake load is very short and in most situations the main effects of the ground shaking are accumulation of down-slope displacements accompanied by a moderate cyclic degradation of soil strength. This means that the focus of seismic slope stability assessments should be on estimating the earthquake-induced deformations, rather than computing a pseudo-static safety factor. This idea was first put forward by Newmark (1965), who proposed a simple 
sliding block model as the analogue for the downslope movement of the soil mass during an earthquake. With this model, one can estimate the permanent earthquakeinduced displacements and assess whether or not they are acceptable.

Nadim et al. (2007) proposed a more rigorous approach for seismic slope stability assessment of clay slopes on the basis of nonlinear, dynamic response analysis. The approach was developed for the evaluation of stability of submarine slopes under earthquake loading, which is one of the most challenging issues in offshore geohazard studies. They recommended that three scenarios should be evaluated and analysed: 1) Failure occurring during the earthquake, where the excess pore pressures generated by the cyclic stresses degrade the shear strength to a level that the slope is not able to carry the static shear stresses; 2) Post-earthquake failure due to an increase in excess pore pressure at critical locations caused by seepage from deeper layers; and 3) Post-earthquake failure due to creep. The advanced numerical methods used for dynamic response analysis provide a reliable picture of the response of a slope during an earthquake, but require detailed information about the geometry, stratification and mechanical properties of the slope.

This paper examines the importance of the effect of permanent shear stresses due to sloping ground, strain softening and sensitivity, ground motion orientation, and the effect of multidirectional shaking on dynamic slope stability analyses. The earthquake-induced permanent shear strains and deformations are used as the metric describing the seismic behaviour of the slope.

\section{Methods}

Effect of permanent shear stresses and strain softening

To investigate the importance of the effect of permanent shear stresses, strain softening, and input motion orientation on the stability of slopes during earthquake shaking, we conducted one-dimensional nonlinear dynamic slope stability analyses in the program QUIVER (Kaynia, 2012). Figure 1 shows the idealized 1D computational model for a slope subjected to earthquake shaking used in QUIVER. The soil profile is modelled by $\mathrm{N}$ shear beam finite elements, each represented by a mass, a spring, and a viscous dashpot, with infinite lateral extensions. A generic soil element has an effective normal stress $\left(\sigma_{\mathrm{n}}\right)$ in the direction normal to the slope, and a consolidation shear stress $\left(\tau_{c}\right)$ acting in the plane of the slope parallel to the dip. The dynamic equation of motion for each node of the system is combined into the global equation of motion, which QUIVER solves in the time domain using the constant acceleration Newmark $\beta$ method (Newmark, 1959). This is an implicit and unconditionally stable integration algorithm method.

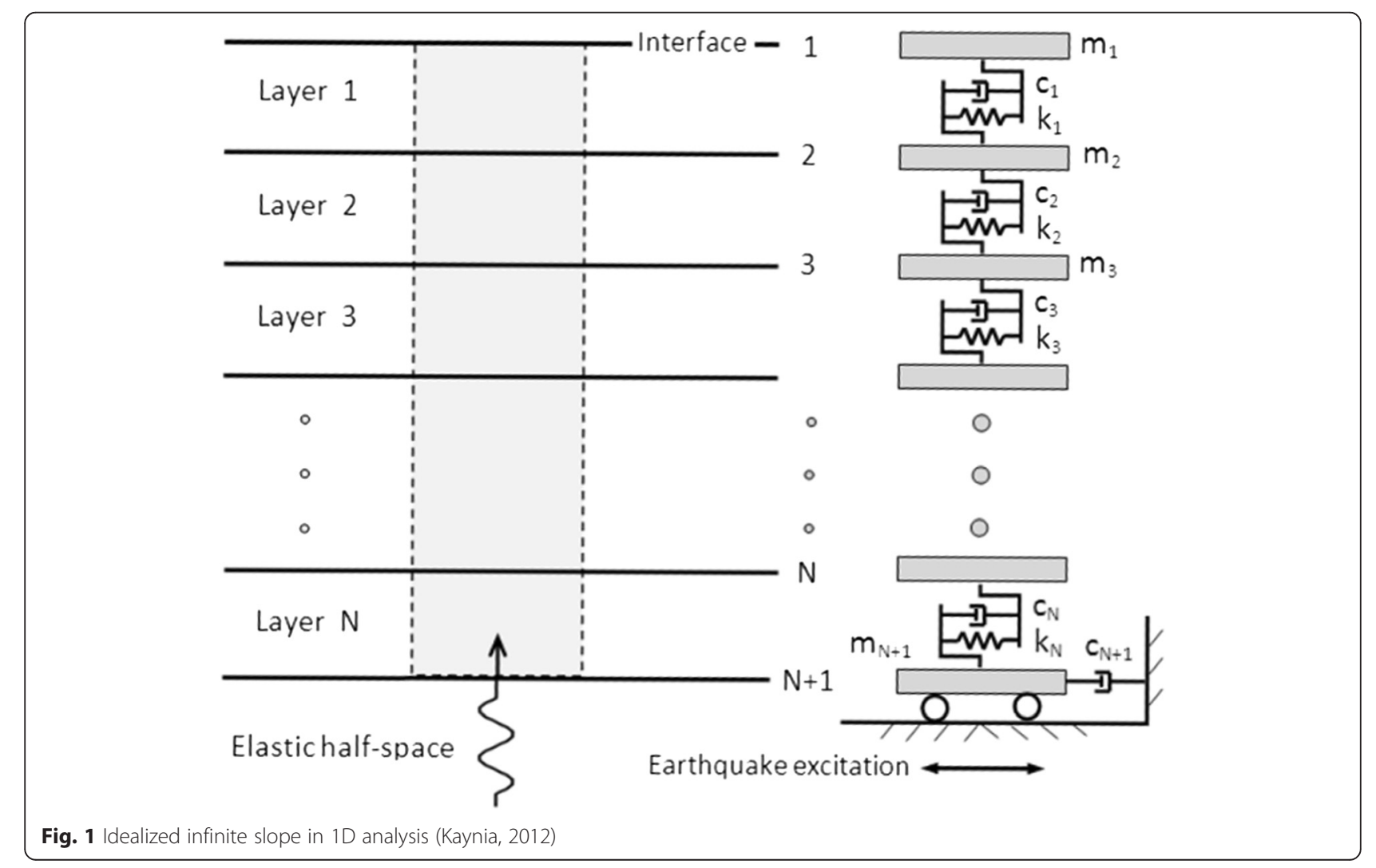


Similar to a 1D seismic site response analysis for flat ground, the earthquake motion is assumed to consist only of shear waves propagating perpendicular to the slope, and those propagating along the plane of the slope (surface waves) are ignored. The earthquake shaking then adds additional cyclic shear stresses acting on the plane of the slope. Figure 2 shows the acceleration time series used in the QUIVER analyses, as well as the corresponding velocity and displacement time series. The acceleration time series is from the PEER strong motion database (Ancheta et al. 2014), and is the north oriented component (azimuth $=0^{\circ}$ ) recorded at the Bursa Tofas site during the 1999 Kocaeli Earthquake $\left(\mathrm{M}_{\mathrm{w}}=7.5\right)$. It is representative of a design ground motion for sites in active crustal regions. In Fig. 2 the positive direction is applied in the downslope direction and the negative corresponds to the upslope direction. Based on the recommendations of Stewart et al. (2008), we performed the dynamic slope stability analyses in QUIVER using the input motion as recorded, with no deconvolution, and with an elastic half-space underlying the soil profile. We scaled the record to a peak ground acceleration (PGA) of $0.2 \mathrm{~g}$.

The soil parameters used in the analyses are modelled after generic soft cohesive soil sites typically found in the offshore environment. Figure 3 shows the site profile. In time domain analyses, the maximum frequency that can be propagated through a soil layer is given by $\mathrm{f}_{\max , \mathrm{i}}=\mathrm{V}_{\mathrm{s}, \mathrm{i}} /(4 \times$ $\left.H_{i}\right)$, where $f_{\text {max }, i}$ is the maximum period that layer $i$ can propagate, $V_{s, i}$ is the shear wave velocity of layer $i$, and $H_{i}$ is the height of layer $i$. Frequencies greater than $f_{\max }$ will not be propagated though the soil layer. We adjusted the thickness of the soil layers so that the maximum frequency propagated through the site was $15 \mathrm{~Hz}$.

The constitutive model implemented in QUIVER consists of a visco-elastic linear loading/unloading response together with strain softening and a kinematic hardening yield function post peak strength. The advantage of QUIVER over other 1D codes is the inclusion of strain softening in the nonlinear soil model. Damping in the loading/unloading cycles is simulated by conventional Rayleigh damping, defined as $C=\alpha \times M+\beta \times K$ where $\mathrm{C}$ is the damping matrix, $\mathrm{M}$ is the mass matrix, $\mathrm{K}$ is the stiffness matrix, and $\alpha$ and $\beta$ are scalar values selected to obtain given damping values for two target frequencies. As recommended by Stewart et al. (2008), we selected the target frequencies as the elastic site frequency, and five times the elastic site frequency. QUIVER was validated against results from 2-dimensional analyses with PLAXIS (PLAXIS 2D Version 9.0, 2009).

\section{Effect of multidirectional shaking}

To investigate the effect of multidirectional shaking on dynamic slope stability analyses, Anantanavanich et al. (2012a) developed the constitutive model MSimpleDSS and implemented it in AMPLE2D, which is an improved version of the finite element code AMPLE2000 (Pestana and Nadim, 2000). The MSimpleDSS model is an effective stress model that includes anisotropy, strain rate adjustable parameters, and allows redistribution of shear stresses, strains, and pore pressures between two directions. The model parameters were estimated
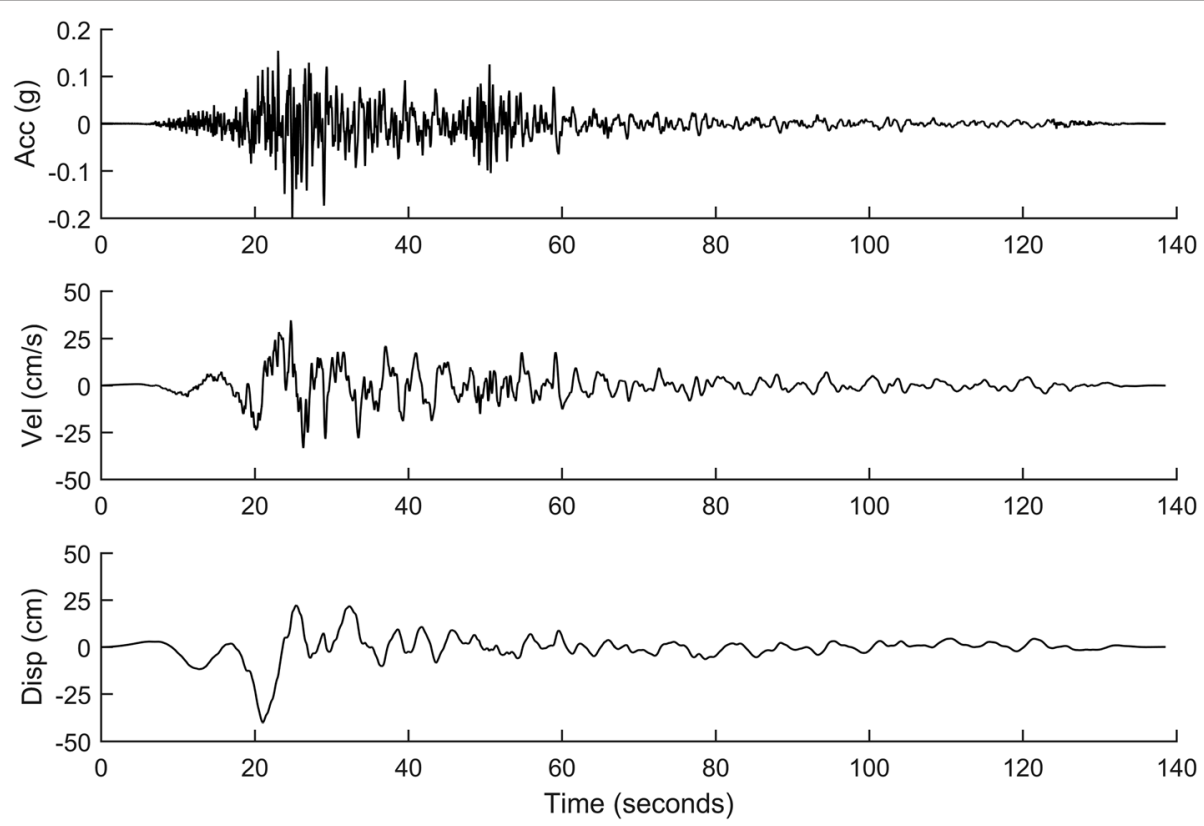

Fig. 2 Ground motion used in site response analyses 


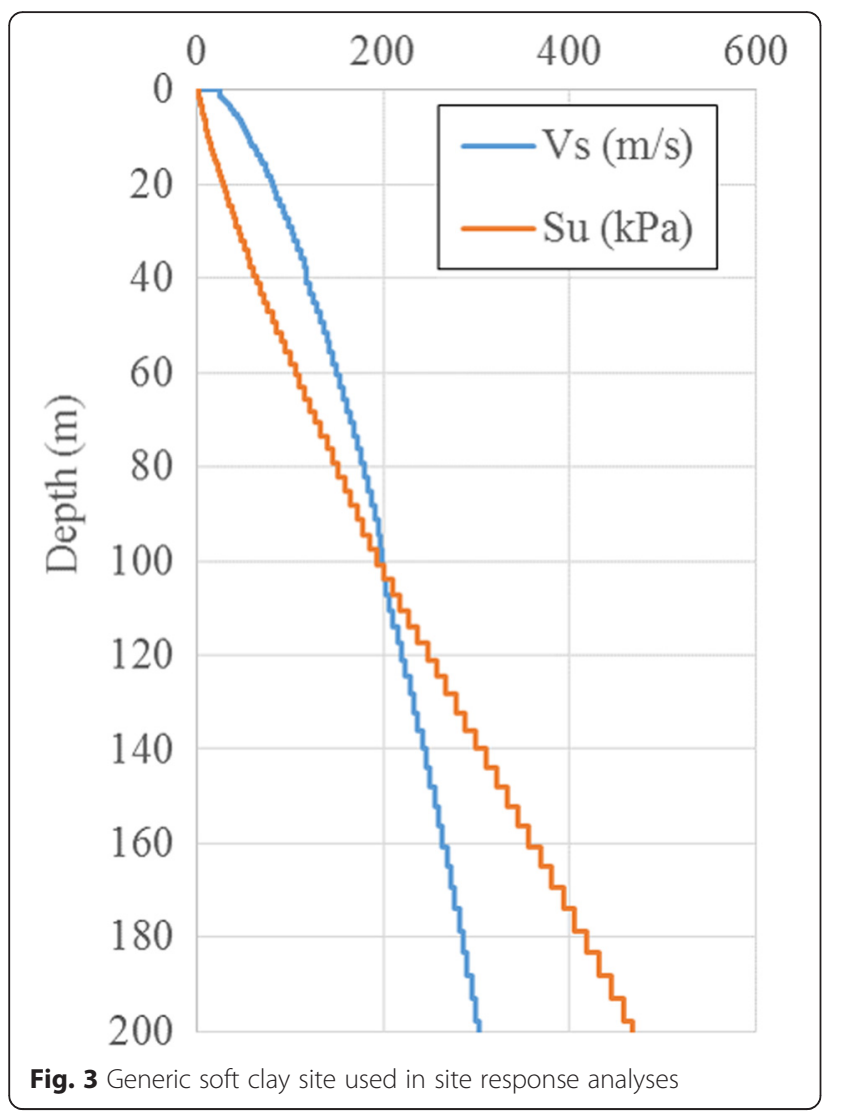

from laboratory tests on normally consolidated Young Bay Mud from the San Francisco Bay, which is representative of medium plasticity soft clay.

\section{Results and discussion}

Permanent shear stresses due to sloping ground

The most important information about the slope geometry is the slope angle. When the ground is sloping a static shear stress develops in the soil. This stress causes the deformations to accumulate in the downhill direction. Biscontin (2001) conducted a series of direct simple shear tests on Young Bay Mud from San Francisco Bay. She observed that the effect of a slope (i.e., a consolidation shear stress) increased the strength of the soil when shearing downslope, but reduced the factor of safety (FoS) for the slope by decreasing the difference between the permanent stress and the soil strength.

Figure 4 displays the effect of slope angle on the response with depth. As the slope angle increases, the transient shear strains and the permanent displacements increase. The PGA decreases as the slope angle increases, except at the surface where the opposite is true. This is because for this scenario the soil yields at about $10 \mathrm{~m}$ depth, with a soil crust riding on top. This is seen in the plot of the transient shear strains, which peak at a depth of $10 \mathrm{~m}$ rather than at the soil surface. In addition, as the slope angle increases, the depth of soil that
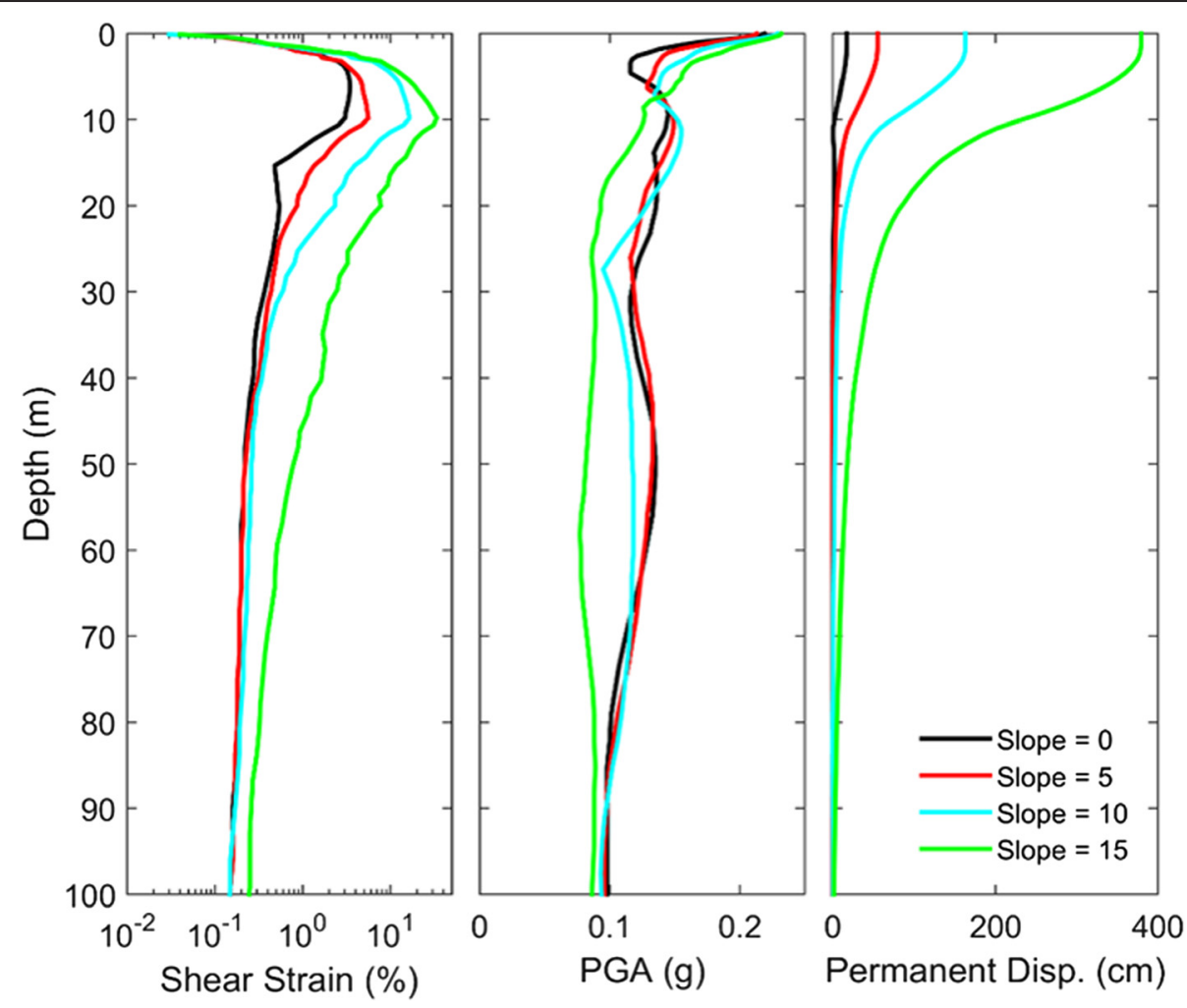

Fig. 4 Effect of slope angle on the seismic response of a soft cohesive soil site 
experiences a permanent displacement increases because the initial static shear strains increase.

We also investigated the effect of slope angle on the response spectra predicted at the soil surface. Figure 5 shows that as the slope angle increases, the energy shifts to longer periods due to the effect of nonlinearity and accumulation of permanent deformation in the downslope direction. The overall amplitude of the response spectra also decreases as the slope angle increases due to an increase in soil damping at larger shear strains.

\section{Strain softening/sensitivity}

Strain softening is the reduction of shear strength as the shear strain increases past the shear strain value where the peak shear strength occurs. Sensitivity $\left(S_{t}\right)$ is defined as the ratio of peak shear strength to the residual shear strength. Soils that have strong strain softening characteristics and high sensitivity are most susceptible to complete failure during earthquake shaking. Strain softening is found for many soils, however, highly sensitive soils are usually clays at high water content $(>0.9 \times$ liquid limit). The most sensitive soils are commonly found in Eastern Canada and Scandinavia. In addition, marine clays may be highly sensitive due to leaching of salt from the pore water, which reduces interparticle bonding. These types of clays are commonly called quick clays.

To investigate the effect of strain softening we performed dynamic slope stability analyses for sensitivity values of $1,1.33,2$, and 4 . Figure 6 presents the four strain softening models used in the analyses. The models peak at a shear stress $(\tau)$ equal to the undrained shear strength $\left(\mathrm{s}_{\mathrm{u}}\right)$ for a given soil layer, then remain flat until a shear strain of $2 \%$ before reducing to the residual strength at a shear strain of $20 \%$. In all of the sensitivity

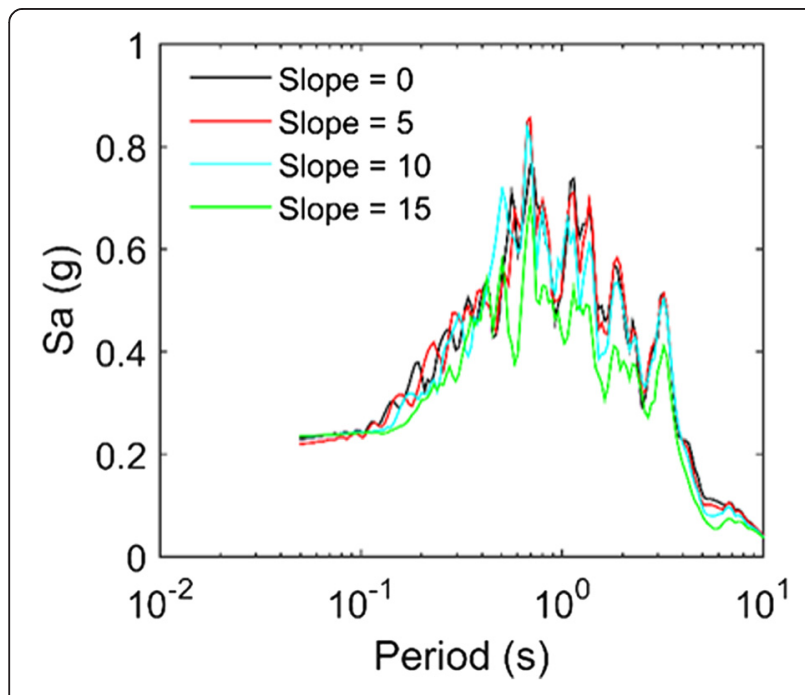

Fig. 5 Effect of slope angle on the response spectra at the soil surface

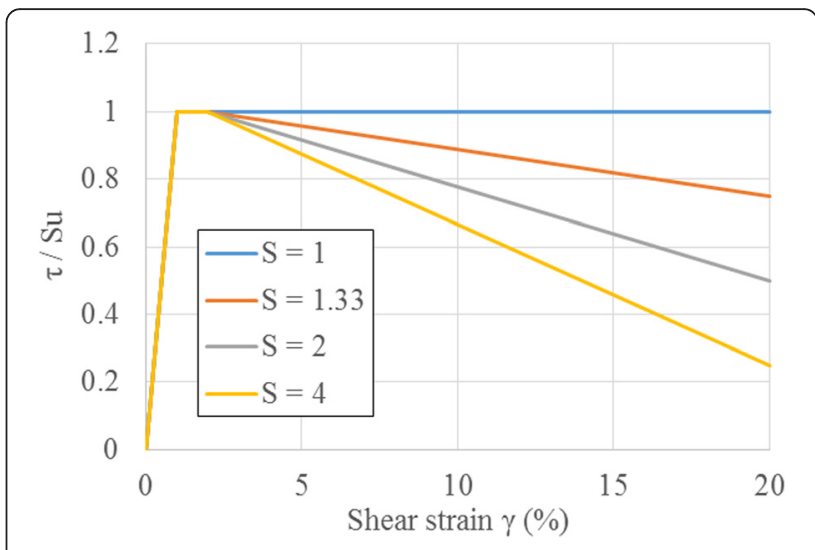

Fig. 6 Strain softening model used in the site response analyses

analyses we used the acceleration time series shown in Fig. 2, the site profile shown in Fig. 3, and slope angles of $0,5,10$, and 15 degrees.

Figure 7 shows the effect of soil sensitivity on the response with depth for a site with a 5 degree slope. For deeper soil layers, where the shear strain remains less than $2 \%$, the results are the same. However, for soils nearer the surface that experience shear strains larger than $2 \%$, more sensitive soils undergo larger shear strains and permanent displacements than less sensitive soils. When the soil sensitivity increases, the depth of soil that experiences a permanent displacement does not increase but remains the same, which is in contrast to the results for when the slope angle increases, as shown in Fig. 4. This is because there is no difference in the soil model until the threshold shear strain of $2 \%$ is reached. Therefore, it is very important to understand the stressstrain characteristics of sensitive soils when including them in slope stability analyses.

In addition to the transient shear strains, the peak ground accelerations, and permanent displacements with depth, we also investigated the difference in the response spectra calculated at the soil surface due to sensitivity. Figure 8 indicates only a small decrease in the response spectral values for the mid-range periods as the soil sensitivity increases. At short periods and long periods the results are the same. This is an interesting result because it indicates that for infrastructure that is more vulnerable to accelerations rather than displacements sensitivity has a negligible effect.

The previous paragraphs looked at the effect of soil sensitivity for only one slope angle. Figure 9 presents a summary of the combined effect of slope angle and soil sensitivity on the maximum permanent displacement. It shows that even a small increase of the slope angle from 0 to 5 degrees increases the maximum permanent displacement from $17 \mathrm{~cm}$ to $55 \mathrm{~cm}$ for the site and ground motion used in this investigation. For a slope angle of 5 


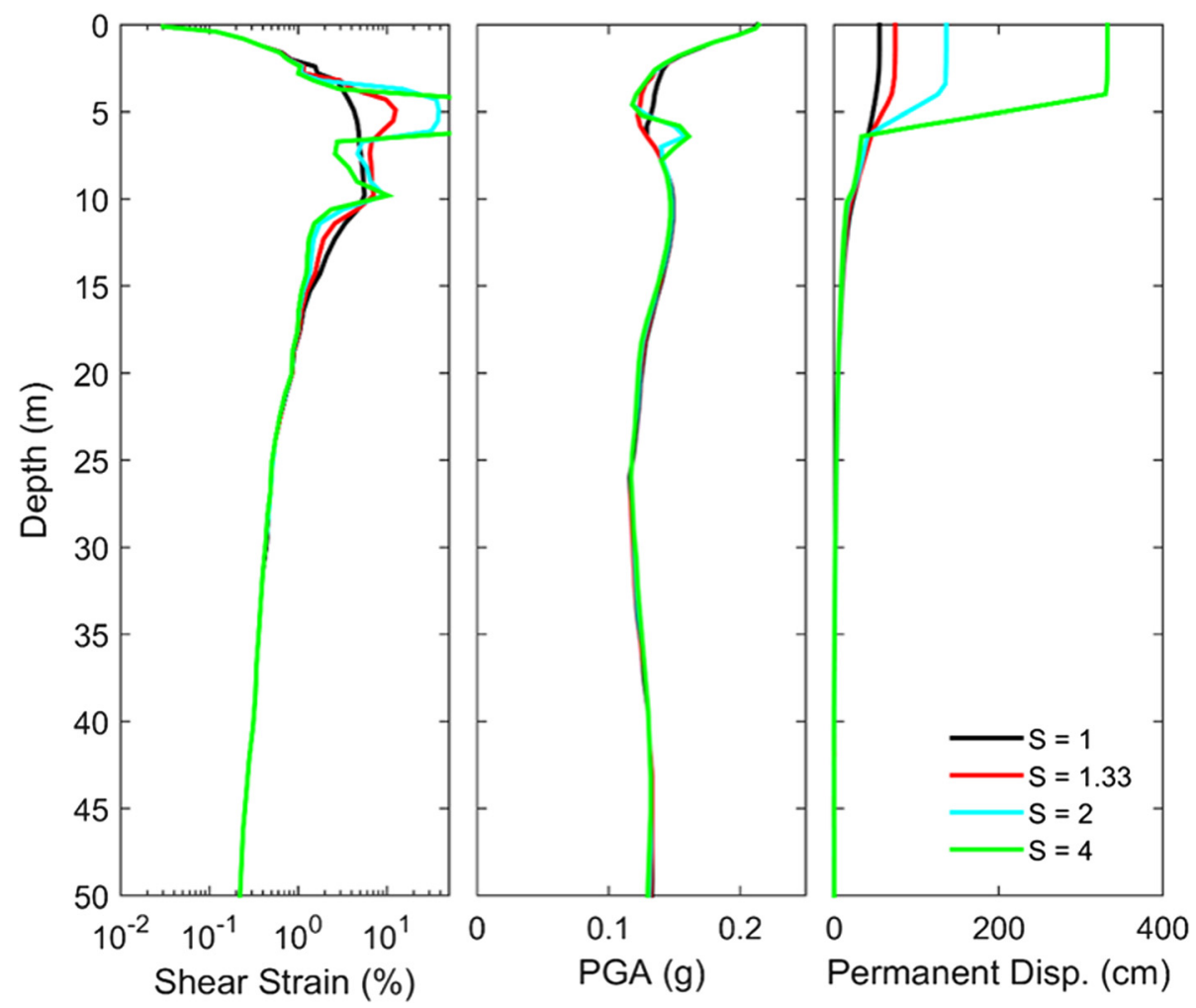

Fig. 7 Effect of soil sensitivity for slope angle of 5 degrees

degrees, changing the soil sensitivity from 1 to 4 increases the maximum permanent displacement from $55 \mathrm{~cm}$ to $330 \mathrm{~cm}$. For a soil with high sensitivity, the mobilized shear strength during the earthquake may fall below the shear stress that is required for static stability. When that happens, the slope essentially fails and the

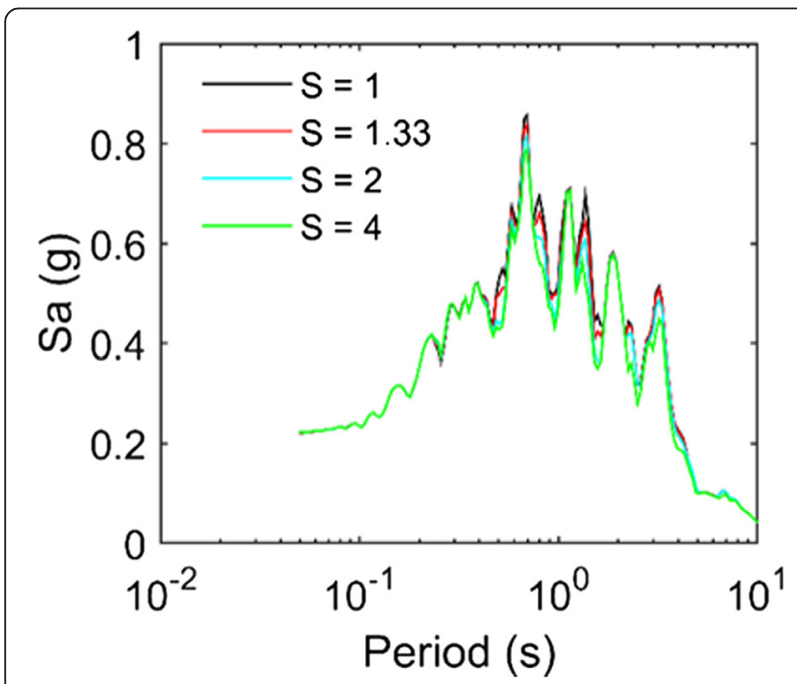

Fig. 8 Effect of soil sensitivity on the response spectra at the soil surface for a slope angle of 5 degrees predicted displacements tend to infinity because there is not enough resistance to stop them. Figure 9 demonstrates the importance of slope angle and soil sensitivity in seismic slope stability analyses.

\section{Orientation of acceleration time series}

Ground motions are often recorded in three orthogonal directions, such as north, east, and up. However, when applying only one of the horizontal components, changing

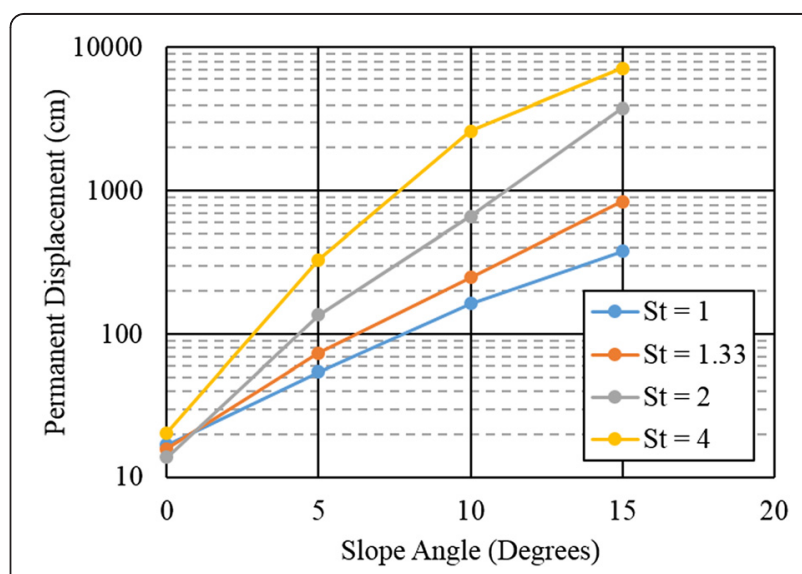

Fig. 9 Maximum permanent displacement compared with slope angle and soil sensitivity 
the orientation can change the intensity and frequency content of the acceleration time series. Therefore, an important consideration when performing dynamic slope stability analyses is the orientation of the acceleration time series with respect to the slope.

In the prior analyses, we applied the north oriented component (azimuth $=0^{\circ}$ ) of the ground motion recorded during the 1999 Kocaeli Earthquake at the Bursa Tofas station. To investigate the effect of the orientation of the acceleration time series, we combined and rotated the two horizontal components to the orientation with the maximum peak ground acceleration (PGA), the orientation with the maximum spectral acceleration value at a period of one second $(\mathrm{Sa}(\mathrm{T}=1))$, and the opposite orientation (azimuth $=180^{\circ}$ ). We then conducted dynamic slope stability analyses in QUIVER using the same site conditions and slope angles as the prior analyses for soil with no sensitivity $\left(S_{t}=1\right)$.

Figure 10 compares the maximum calculated permanent displacements for the four different orientations and slope angles. The given orientation is the same as shown in Fig. 9 for $S_{t}=1$. If the opposite orientation is applied (i.e. the negative direction in Fig. 2 is downslope and positive is upslope), then the calculated permanent displacements are larger. This is because the ground motion has larger displacements in the negative direction than the positive direction, as shown in Fig. 2. Applying the orientation with the maximum PGA gives permanent displacements between those predicted using the given and opposite orientation. The orientation with the maximum spectral acceleration at a period of one second gives the largest permanent displacements for slope angles of 5, 10 and 15 degrees. This is because longer periods are more correlated with displacements than shorter periods, such as PGA.

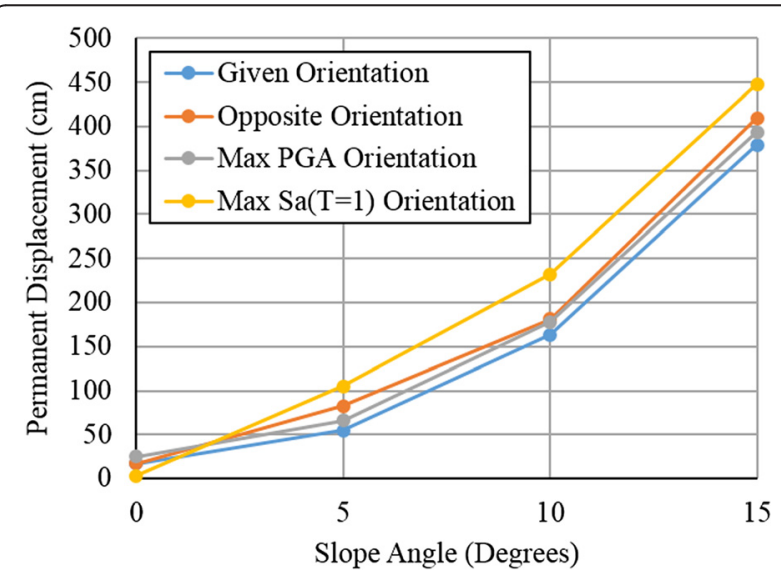

Fig. 10 Comparison of the effect of ground motion orientation on the maximum calculated permanent displacement

\section{Multidirectional shaking}

One-dimensional models provide a good approximation of the seismic behaviour of slopes for depths that are relatively small compared to the slope length. This is often the case for offshore submarine landslides, where the ratio between thickness and length of the sliding mass is relatively small. However, for more complicated offshore scarps or for onshore mountainous regions, deep seated failures often develop along curved surfaces, and the top and bottom slope boundaries have a significant effect. Slopes with level top and bottom boundaries will have smaller permanent displacements than their corresponding 1D infinite slope approximation due to the reduced consolidation shear stresses on the level ground boundaries and because the bottom boundary will act as a buffer. In addition, slope failures along curved sliding surfaces in $2 \mathrm{D}$ models require more energy than flat surfaces predicted in 1D models, which leads to smaller permanent displacements. For complicated topographies, 3D analyses may even be necessary due to wave refraction at the edges and wave interferences.

Even when modelled in 2D or 3D, most seismic slope stability analyses only consider one component of the ground motion, when in reality ground shaking occurs in all directions. Kammerer et al. (2005) performed extensive laboratory stress-controlled cyclic tests on granular soil, and found that the soil response under multidirectional shearing tended to generate pore pressure faster than that of unidirectional shearing. Su and Li (2003) applied both unidirectional and multidirectional shaking to level saturated sand deposits in a centrifuge and found that the maximum pore pressure at great depths for multidirectional shaking was about $20 \%$ larger than that in one-directional shaking and the difference reduced to about $10 \%$ near the surface.

Using the MSimpleDSS model in AMPLE2D, Anantanavanich et al. (2012b) performed nonlinear dynamic slope stability analyses for two generic soft clay sites with depths of $20 \mathrm{~m}$ and $100 \mathrm{~m}$, and four sets of ground motions. They compared the estimated permanent displacements and excess pore pressures generated from applying one or both horizontal components of a ground motion at the same time for different slope angles.

The effect of multidirectional shaking on displacement is expressed in terms of the multidirectional displacement ratio (MDR), which is the percent increase in displacement accumulated under multidirectional shaking over that of one directional shaking at a given depth. Figure 11 shows the average value of MDR for the four ground motions investigated for the $20 \mathrm{~m}$ and $100 \mathrm{~m}$ soil profiles with a slope angle of 10 degrees. For both soil profiles, multidirectional shaking predicts smaller permanent displacements for the bottom half, and larger 


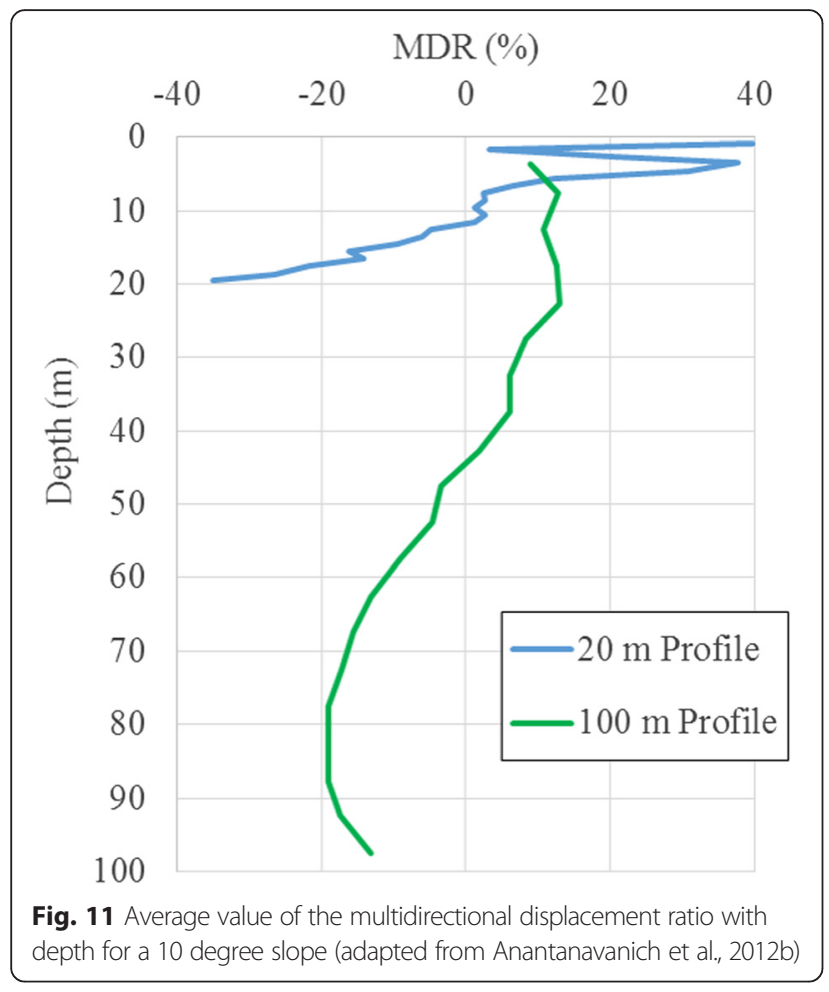

Fig. 11 Average value of the multidirectional displacement ratio with
depth for a 10 degree slope (adapted from Anantanavanich et al., 2012b) permanent displacements for the upper half of the profile than unidirectional shaking. At the surface, the $20 \mathrm{~m}$ and $100 \mathrm{~m}$ profiles predict about a $40 \%$ and $20 \%$ increase in permanent displacement for multidirectional shaking over unidirectional shaking, respectively.

Similar to the MDR, the effect of multidirectional shaking on the excess pore pressure is expressed in terms of the multidirectional pore pressure ratio (MPR), which is the percent increase in the magnitude of pore pressure developed under multidirectional shaking over that of one-directional shaking at a given depth. Figure 12 presents the average value of MPR for the four ground motions investigated for the $20 \mathrm{~m}$ and $100 \mathrm{~m}$ soil profiles with slope angles of 0,5 , and 10 degrees. The $100 \mathrm{~m}$ deep soil profile shows that multidirectional shaking causes a $30 \%$ increase in pore pressure generation near the bottom of the profile, which reduces to $0 \%$ at around a depth of $10 \mathrm{~m}$ before increasing again to $10 \%$ near the surface. These results are similar to those found by Su and $\mathrm{Li}$ (2003). The excess pore pressures generated at depth by multidirectional shaking could propagate to the surface and cause failure long after earthquake shaking has stopped. The slope angle appears to have a negligible effect on excess pore pressure generation. For the $20 \mathrm{~m}$ soil profile, the MPR value varies from $20 \%$ to $40 \%$ over all depths. Based on these results, unidirectional site response analyses could give unconservative results.

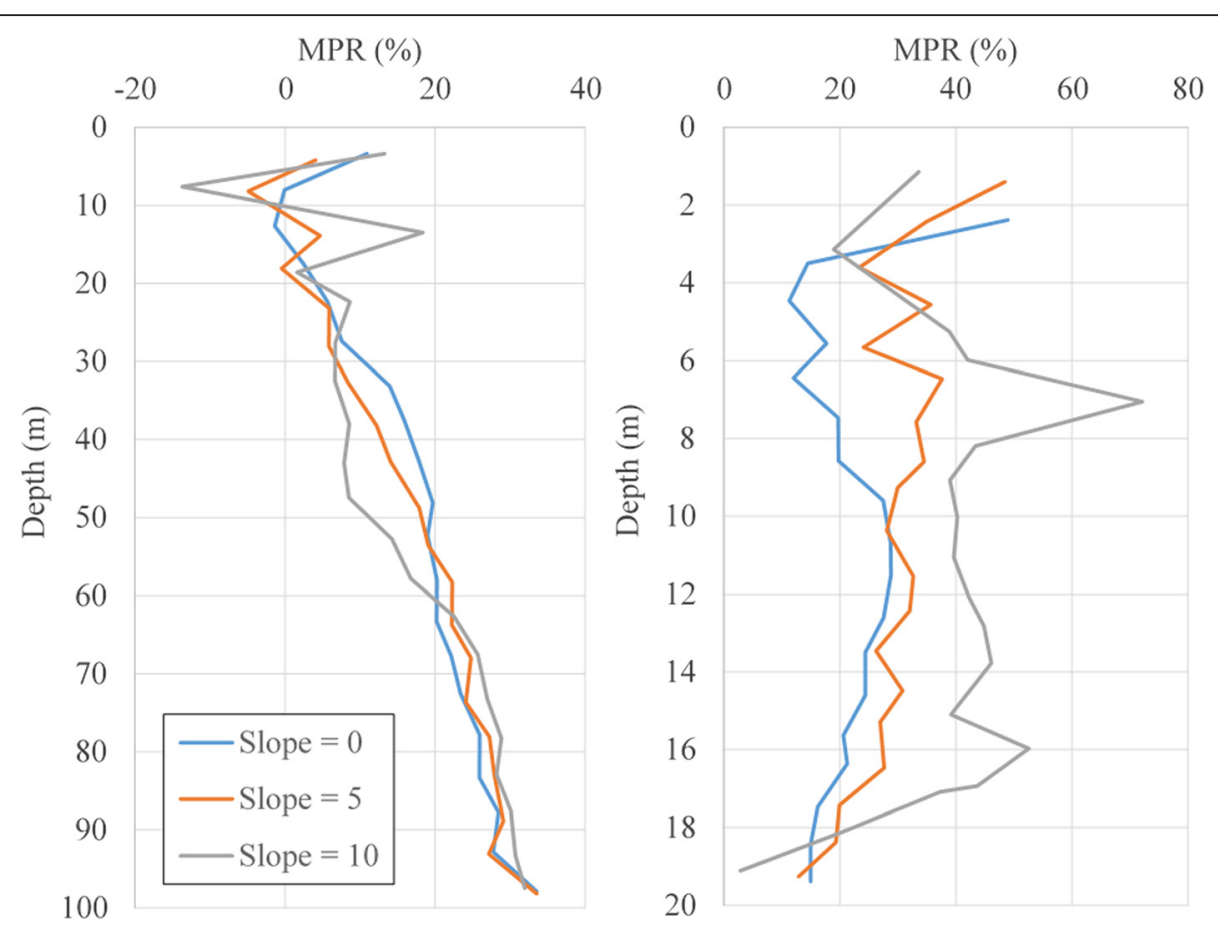

Fig. 12 Average value of the multidirectional pore pressure ratio (MPR) for different slope angles (adapted from Anantanavanich et al., 2012b) 


\section{Conclusions}

The numerical results from the site response analysis programs QUIVER and AMPLE2D show that permanent shear stresses due to sloping ground, strain softening and sensitivity, ground motion orientation, and multidirectional shaking all have a large influence on the permanent displacements estimated from earthquakeinduced landslide hazard analyses.

For the analyses conducted in this investigation, increasing the slope angle by 10 degrees or increasing the sensitivity from 1 to 4 for non-zero slopes resulted in an order of magnitude difference in the predicted permanent displacements. Changing the orientation of the ground motion from the as given orientation to the orientation with the maximum spectral acceleration value at a period of 1 second increased the estimated permanent displacements by $50 \mathrm{~cm}$ or more for all non-zero slopes. Applying two horizontal ground motion components instead of only one resulted in 10-40\% larger permanent displacements than unidirectional shaking near the soil surface. In addition, multidirectional shaking also predicted larger excess pore pressures than unidirectional shaking.

We also found that as the slope angle increases, the energy in the response spectra predicted at the soil surface shifts to longer periods and the overall amplitude decreases. However, soil sensitivity has a negligible effect on the response spectra calculated at the soil surface.

The results from this investigation show that it is important for site investigations to provide adequate information to model the correct slope angle and soil sensitivity. In addition, project managers should consider the ground motion orientation when selecting acceleration time series for analysis. Finally, analyses with only one ground motion component may give unconservative results, therefore, multidirectional analyses should be conducted when possible.

\section{Competing interests}

The authors declare that they have no competing interests.

\section{Authors' contributions}

BC performed the Quiver analyses, participated in the MSimpleDSS analyses, and prepared the manuscript. AMK and FN provided technical support, feedback, and comments at all stages of the research. All authors read and approved the manuscript.

Received: 14 October 2015 Accepted: 12 May 2016

Published online: 20 May 2016

\section{References}

Anantanavanich, T., Pestana, J., and Carlton, B. 2012a. Development of a Constitutive Model to Describe the Dynamic Response of Soft Submarine Clay Deposits. In: 15th World Conference on Earthquake Engineering, Lisbon, Portugal, 24-28 September 2015.

Anantanavanich, T., Pestana, J., and Carlton, B. 2012b. Multidirectional Site Response Analysis of Submarine Slopes Using the Constitutive Model MSimpleDSS. In: 15th World Conference on Earthquake Engineering, Lisbon, Portugal, 24-28 September 2015.
Ancheta, T.D., R.B. Darragh, J.P. Stewart, E. Seyhan, W.J. Silva, B.S.J. Chiou, K.E. Wooddell, R.W. Graves, A.R. Kottke, D.M. Boore, T. Kishida, and J.L. Donahue. 2014. NGA-West2 Database. Earthquake Spectra 30: 989-1005.

Biscontin, G. 2001. Modeling the dynamic behavior of lightly overconsolidated soil deposits on submerged slopes. Berkeley: Dissertation, University of California.

Collins, B.D., and Jibson, R.W. 2015. Assessment of existing and potential landslide hazards resulting from the April 25, 2015 Gorkha, Nepal earthquake sequence. U.S. Geological Survey Open-File Report 2015-1142, p. 50.

Kammerer, A., Pestana, J., and Seed, R. 2005. Behavior of Monterey 0/30 sand under multidirectional loading conditions. In Yamamuro, J.A., and Koseki, J. (eds) Geomechanics: Testing, Modeling, and Simulation. Geotechnical special publication 143, ASCE.

Kaynia, A.M. 2012. QUIVER_slope: Numerical code for one-dimensional seismic response of slopes with strain softening behavior. NGI Rapport 2007185100-79-R, pp 22

Nadim, F., Biscontin, G., and Kaynia, A.M. 2007. Seismic Triggering of Submarine Slides. In: Offshore Technology Conference'07, Houston, Texas, paper OTC 18911.

Newmark, N.M. 1965. Effects of Earthquakes on Dams and Embankments. Geotechnique 15(2): 139-160.

Newmark, N.M. 1959. A method of computation for structural dynamics. Journal of the Engineering Mechanics Division, EM 8(3): 67-94.

Pestana, J.M., and F. Nadim. 2000. Nonlinear site response analyses of submerged slopes. Geotechnical Engineering Report UCB/GT/2000-04. Berkeley: University of California. PLAXIS 2D Version 9.0. 2009. User Manual. The Netherlands: PLAXIS, Delft.

Stewart, J.P., Kwok, A.O., Hashash, Y.M.A, Matasovic, N., Pyke, R., Wang, Z., and Yang, Z. 2008. Benchmarking of Nonlinear Geotechnical Ground Response Analysis Procedures. Pacific Earthquake Engineering Research Center Report 2008/04.

Su, D., and X. Li. 2003. Centrifuge tests on earthquake response of sand deposit subjected to multi-directional shaking. In 16th ASCE Engineering Mechanics Conference. Seattle: University of Washington.

\section{Submit your manuscript to a SpringerOpen ${ }^{\circ}$ journal and benefit from:}

- Convenient online submission

- Rigorous peer review

- Immediate publication on acceptance

- Open access: articles freely available online

- High visibility within the field

- Retaining the copyright to your article

Submit your next manuscript at $>$ springeropen.com 